\title{
EDITORIAL: ANALOG AND MIXED-SIGNAL IC DESIGN AND DESIGN METHODOLOGIES
}

During the past decades, we have witnessed a progressive substitution of analog-based solutions by digital ones, in parallel with the continuous evolution of technological processes, especially CMOS, towards deep submicron technologies. This technological shrinkage has enabled the integration of more and more functionalities on a single chip, with the consequent reduction in area, cost and power consumption; therefore, fueling, for instance, the information technology revolution.

Although analog circuitry has been, in most cases, relegated to the interface between the digital processing subsystems and the analog world, e.g., the physical channel in communication systems, the trend towards higher system integration is also reaching the analog and mixed-signal circuitry. Nowadays, it is not uncommon to integrate complex digital subsystems like microprocessors, memories, etc., with analog and mixed-signal blocks, RF blocks and even sensors onto the same substrate, constituting what is already commonly known as a System-on-Chip (SoC).

New applications and higher system functionalities are continuously demanding more aggressive specifications of analog and mixed-signal circuits in hostile environments (i.e., continuous decrease of supply voltages, substrate coupling with noisy signals, etc.) and it is usually the performance of this circuitry which limits the performance of the overall system. Demands also extend to design methodologies and tools for analog and mixed-signal subsystems, traditionally much less developed than for digital circuits. The comparatively low number of CAD tools and methods may dramatically increase the design time and cost of a SoC product, and it may eventually be responsible for the loss of the market window.

In this context, the extension of the scope of Integration-the VLSI journal to cover the analog and mixed-signal field was a natural evolution. Hopefully, this Special Issue inaugurates a permanent presence of papers on analog and mixed-signal design. The call-for-papers had a wide acceptance and numerous submissions were received. All papers were subject to peer-review by international experts and it was a tough work to select those which would be included in the limited number of pages of the special issue. I hope that you will enjoy these papers.

Regarding the organization of the issue, six papers have been selected dealing with significant aspects of design and design methodologies of analog and mixed-signal ICs. Operational amplifiers are the most common block in analog design. Analog designers have to face continuously decreasing supply voltages due to the scaling down towards deep submicron IC technologies. However, transistor threshold voltages are not decreasing at the same rate, which seriously compromises to obtain similar performances. The first paper, by Carrillo et al., deals with operational amplifier design for extremely low supply voltages. Novel dynamic input level shifters allow to extend the input commonmode range from rail to rail while keeping the transconductance approximately constant. The second paper, by Lotfi et al., focuses on the reduction of power consumption and proposes a low-power architecture for high-speed operational amplifiers in switched-capacitor applications. 
Bipolar transistor circuits are nowadays the technology of choice for the highspeed processing needs in some applications like wireless communication systems. The performance of some blocks of these systems strongly depends on the D-latches composing them. The third paper, by Alioto et al., deals with the performance evaluation of the low-voltage CML D-latch. An analytical model of the low-voltage CML latch is presented and this topology is compared to the conventional one in terms of delay and power-delay trade-off.

Data converters are the key blocks in the interface between the analog and the digital world. In parallel with the ever-increasing complexity and performance of digital cores, higher performances are also demanded from data converters, in terms of accuracy, speed, power consumption and area occupation. In the fourth paper, Quinn and Pribytko propose a new architecture of the 1.5 bit stage found in algorithmic and pipeline analog-to-digital converters. This architecture allows to achieve high linearity without needing precision capacitors.

Explosive growth of communications has been a main force driving the development of electronic equipments. Among the different physical media, electrical power lines arise as an attractive communication alternative mainly due to the ubiquitous infrastructure. To this end, in the fifth paper, Guerra et al. address the design of a CMOS mixed-signal modem ASIC for data transmission over the low-voltage power-line network.

Analog layout automation is far from its digital counterpart. Reported approaches usually fall into one of these two classes: (a) optimization-based approaches, which formulate the device placement and routing as an optimization problem and (b) template-based approaches, which store designer experience on the layout of specific blocks. The first group of methods is general but fails to incorporate expertise, which may impact performance and, even more important, hits against skeptic analog designers. The second group of approaches gains in speed and incorporation of layout knowledge at the price of smaller flexibility and a relatively high cost of template creation. In the sixth paper, Jangkrajarng et al. advance in palliating some drawbacks of the second group of approaches by proposing a method which automatically extracts templates from existing layouts.

I am becoming close to the end of this Editorial note and it is already time to thank so many people who contributed to make this Special Issue possible. First, my gratitude to Prof. Georges Gielen, from Katholieke Universiteit Leuven, for his support and encouragement. Thanks, Georges, for the invitation to launch this initiative. I would also like to express my gratitude to the authors for the time and effort put in transmitting their ideas and to all other authors who submitted highly valued contributions but whose papers could not be selected. Thanks also to the many reviewers who provided detailed comments on the submitted papers and without whose expertise this Special Issue would not have been possible.

Last, but not least, I would like to thank Mr. Steven Watson, for his permanent support and enthusiasm, so many people at the Elsevier Technical Support Team for their patience and willingness to solve all problems with the electronic submission system, and Mrs. Chris Mertens (KUL) for her assistance in handling the reviewers' evaluations.

Francisco V. Fernández

Institute of Microelectronics of Seville, IMSE-CNM-CSIC, 41012 Seville, Spain 
